\title{
Differentiation of Low-Grade and High-Grade Glioma Using the Combination of Conventional Magnetic Resonance Imaging and Apparent Diffusion Coefficient Value
}

\author{
Rahmad Mulyadi, ${ }^{1, *}$ Mochammad Hatta ${ }^{2}$, Andi Asadul Islam ${ }^{3}$, Bachtiar Murtala ${ }^{4}$, \\ Jumraini Tammase ${ }^{5}$, Reninda Ananda Aman ${ }^{6}$, Eka Susanto \\ ${ }^{1}$ Neuroradiology Division, Department of Radiology, Faculty of Medicine, Universitas Indonesia - Cipto Mangunkusumo National Central \\ General Hospital, Jl. Diponegoro No.71, Jakarta Pusat, Jakarta, Indonesia \\ ${ }^{2}$ Molecular Biology and Immunology Laboratory, Faculty of Medicine, Universitas Hasanuddin, Jl. Perintis Kemerdekaan KM 10, Makassar, Indonesia \\ ${ }^{3}$ Department of Neurosurgery, Faculty of Medicine, Universitas Hasanuddin, Jl. Perintis Kemerdekaan KM 10, Makassar, Indonesia \\ ${ }^{4}$ Department of Radiology, Faculty of Medicine, Universitas Hasanuddin, J1. Perintis Kemerdekaan KM 10, Makassar, Indonesia \\ ${ }^{5}$ Department of Neurology, Faculty of Medicine, Universitas Hasanuddin, Jl. Perintis Kemerdekaan KM 10, Makassar, Indonesia \\ ${ }^{6}$ Department of Neurosurgery, Faculty of Medicine, Universitas Indonesia - Cipto Mangunkusumo National Central General Hospital, \\ Jl. Diponegoro No.71, Jakarta Pusat, Jakarta, Indonesia \\ ${ }^{7}$ Department of Anatomical Pathology, Faculty of Medicine, Universitas Indonesia - Cipto Mangunkusumo National Central General Hospital, \\ Jl. Diponegoro No.71, Jakarta Pusat, Jakarta, Indonesia \\ *Corresponding author. E-mail: dr_rahmad_radiologi@yahoo.com
}

Received date: Oct 9, 2019; Revised date: Jan 15, 2020; Accepted date: Jan 21, 2020

\section{Abstract}

B ACKGROUND: The conventional magnetic resonance imaging (cMRI) and apparent diffusion coefficient (ADC) may have a role in predicting tumor grade for gliomas and may in turn assist in identifying tumor biopsy sites. In this study, we aimed to determine the added value of a joint approach of diffusion-weighted imaging (DWI) and cMRI to determine of low grade and high-grade glioma, compare to cMRI alone.

METHODS: Data were collected from 56 glioma patients, who underwent examinations and received treatment at Cipto Mangunkusumo National Central General Hospital, Jakarta, Indonesia, from the period of 2015-2018. Inclusion criteria was patients who underwent cMRI with a DWIADC sequence and patient that were diagnosed with glioma according to the histopathological results. Pathology reports of the imaging results were reviewed independently.
A receiver operator curve (ROC) analysis assessed the predictive potential of cMRI and ADC values for low-grade and high-grade gliomas.

RESULTS: Fifty-six subjects met the inclusion criteria. The combination of MRI and ADC values increased sensitivity (to $90 \%$ ) and negative predictive value (to $92.9 \%$ ), and also improved the negative likelihood ratio (to 0.14). However, the combination of MRI and ADC values had the highest area under the curve $(78.6 \%)$ and sensitivity $(78.6 \%)$, which was similar to the separated examination.

CONCLUSION: The combination of ADC value and conventional MRI increases sensitivity in differentiating low-grade and high-grade glioma compared to separated examination.

KEYWORDS: glioma, conventional MRI, ADC value

Indones Biomed J. 2020; 12(1): 69-77

\section{Introduction}

Gliomas are the most common primary brain tumors in adults. Glial tumors are made up of astrocytomas, oligodendrogliomas, mixed oligo-astrocytic, and mixed glioneuronal tumors, which arise from the supporting glial cells of the central nervous system (CNS).(1) According to the Central Brain Tumor Registry of the United States (CBTRUS) Statistical Reports, there were 392,982 cases of 
brain tumors reported from 2011 to 2015. Gliomas account for $26 \%$ of all primary brain tumors and $81 \%$ of malignant CNS tumors.(2) Glioma grading has an important role in treatment strategies because high-grade gliomas after resection are treated with adjuvant radiation therapy or chemotherapy, while low-grade gliomas are not. $(3,4)$ To diagnose glioma, histopathological examination remains the gold standard. Based on histopathologic characteristics such as cytological atypia, anaplasia, mitotic activity, microvascular proliferation and necrosis, The World Health Organization (WHO) classification system categorizes gliomas from grade I through grade IV.(1) However, some diagnostic difficulties arise from sampling and overlapping features leading to an underestimation of tumor grade, especially for high-grade lesions. These limitations are motivating research into non-invasive imaging techniques.(5-7)

As for imaging, conventional contrast-enhanced magnetic resonance imaging (MRI) is the gold standard non-invasive technique.(5,6) However, the classification and grading of gliomas with conventional MRI is reportedly unreliable due to its sensitivity, which ranges from $55.1 \%$ to 83.3\%.(8)

In a previous study, it is stated that the apparent diffusion coefficient (ADC) tumor value provides more adequate data than conventional MRI, reflecting a suitable histopathological grade.(9) With the advent of technology, multiparametric MRI can demonstrate physiology and molecular properties of the brain with the ADC.(10) Also, multiparametric MRI increases the accuracy of brain tumor diagnoses.(11) Several studies have reported a correlation between tumor and ADC values. $(9,12,13)$ The ADC sequence is based on water molecules diffusion within the tissue. Normal and diseased brain areas have different ADCs because of the brain microarchitecture heterogeneity. The higher diffusion of brain tissue, the higher the value of ADC. Therefore, the ADC value can identify issues quantitatively. (7) If ADC can predict cellularity and tumor grade, ADC value combined with conventional MRI will likely be useful in improving prediction of glioma grade. In this study, by combining cMRI and ADC imaging, it is expected to increase the diagnostic value comparing to cMRI alone, and it can help in determining the next therapeutic action.

\section{Methods}

This study has obtained institutional review board approvals from the Health Research Ethics Committee,
Faculty of Medicine, Universitas Indonesia (No. 0529/ UN2.F1/ETIK/2018) and the Health Research Ethics Committee Cipto Mangunkusumo National Central General Hospital (No. LB.02/2.2.1/0585/2018), for the cMRI and Anatomic Pathology patient data collection at Cipto Mangunkusumo National Central General Hospital, Jakarta, Indonesia.

\section{Study Design}

This was a retrospective analytic study, conducted between 2015-2018. A diffusion-weighted imaging (DWI)-ADC data from head MRI were obtained, and their association with low- and high-grade gliomas, based on histopathological examination, were investigated to evaluate the relationships between data.

Two-hundred-ninety-two patients with a clinical susceptibility of space-occupying lesion underwent examinations and received treatment at Cipto Mangunkusumo National General Hospital. Inclusion criteria was patients who underwent cMRI with a DWI$\mathrm{ADC}$ sequence and patient that were diagnosed glioma according to histopathological results. Exclusion criteria was patient that were not diagnosed glioma according to histopathological results. Patients whose diagnosis was not a brain tumor based on MRI and glioma based on histopathology, or who had incomplete examination results or no tumor were excluded. As much as 236 patients met exclusion criteria with the majority classified as meningioma $(\mathrm{n}=96)$ and hypophyseal adenoma $(\mathrm{n}=51)$, followed by sellar region tumor $(\mathrm{n}=16)$, embryonal tumors $(n=14)$, metastatic tumors $(n=13)$, schwannoma $(n=12)$, lymphomas $(\mathrm{n}=6)$, neuronal and mixed neuronal-glial tumor $(n=5)$, ependymal tumors $(n=4)$, germ cell tumors $(n=4)$, mesenchymal tumors $(n=3)$, choroid plexus tumors $(n=3)$, and other lesions which were not classified in the WHO Classification of Tumors of the Central Nervous System $2016(\mathrm{n}=9)$.

\section{Materials}

All patients underwent MRI using MRI Siemens 1.5 Tesla Avanto (Siemens, Berlin, Germany). MRI images include: T2-weighted image (T2WI): (time repetition (TR)/time echo (TE) 5160/112 ms, section thickness $5 \mathrm{~mm}$; intersection gap $1 \mathrm{~mm}$; matrix $269 \times 384$; field of view (FOV) $20.1 \mathrm{~cm} \times$ $23.0 \mathrm{~cm}$ ). T1-weighted image (T1WI): (TR/TE 500/9.4 ms, section thickness $5 \mathrm{~mm}$; intersection gap $1 \mathrm{~mm}$; matrix 256 $\times 256$; FOV $23.0 \mathrm{~cm} \times 23.0 \mathrm{~cm}$ ). T1WI was performed in all patients. T2-weighted-Fluid-Attenuated Inversion Recovery (T2 FLAIR) (TR/TE 7000/92 ms; inversion time 2214.1 
$\mathrm{ms}$; section thickness $5 \mathrm{~mm}$; intersection gap $1 \mathrm{~mm}$; matrix $230 \times 256$; FOV $23.0 \mathrm{~cm} \times 23.0 \mathrm{~cm}$ ). DWI was obtained in the axial plane (TR/TE 4000/97, section thickness $5 \mathrm{~mm}$, intersection gap 1, matrix $128 \times 128$; FOV $23.0 \mathrm{~cm} \times 23.0$ $\mathrm{cm})$.

DWIs were obtained with $b$ values of 0,500 , and 1000 $\mathrm{mm}^{2} / \mathrm{s}$. The ADCmin value was determined by placing the regions of interest (ROI) using a workstation (Syngo MR Workplace, Munich, Germany); the placement of five ROI spots was done by one researcher. For DWI identification, tumors with hyperintense lesions were chosen, whereas tumors with a hypointense lesion were identified for ADC measurement. ROI placement was done carefully with regard to the visual image of the tumor, for an area of 1.6 $\mathrm{mm}^{2}$. After that, the lowest value for ADC in $\mathrm{mm}^{2} / \mathrm{s}$ was obtained.

\section{Glioma Diagnosis}

Glioma diagnosis was based on histopathological criteria according to the WHO Classification of Tumors of the Central Nervous System 2016.(14) Tumors were assessed as WHO grade I, II, III, and IV. The morphology codes for malignancy were based on the International Classification of Diseases for Oncology (ICD-O) [742A]. For statistical purposes, WHO grade I and II tumors were grouped as lowgrade tumors, while WHO grade III and IV tumors were grouped as high-grade tumors.

\section{MRI Scoring}

The Dean criteria were used for conventional MRI scoring by evaluating midline shift, presence of edema, tumor hemorrhage, tumor border, presence of cyst and necrotic tissue, and presence of a mass effect. Each of these points was given a weighing score of $0-2$, with an overall minimum score of zero and maximum score of 14 .

\section{Statistical Analysis}

Brain tumor diagnoses were found in the medical records, MRI and DWI-ADC results, and histopathological results. Data were then analyzed using the Statistical Package for the Social Sciences (SPSS) version 20 (IBM Corporation, New York, USA), and the cut-off point of each variable was determined using Microsoft Excel (Microsoft, Washington, USA) to differentiate low- and high-grade gliomas. Sensitivity, specificity, accuracy, positive predictive value (PPV), negative predictive value (NPV), positive likelihood ratio (LR+), and negative likelihood ratio (LR-) were calculated.

\section{Results}

This was an analytical study with a cross-sectional design from the MRI data of brain tumor patients, with ADCmin and ADCmax. Fifty-six patients with gliomas were included in the statistical analyses. The conventional MRI scans of low grade and high-grade gliomas were demonstrated in Figure 1 and Figure 2. The histopathological findings that provided a diagnosis were demonstrated in Figure 3.

\section{Characteristics of Subjects}

In this study, the low-grade and high-grade glioma proportions were equal (50\%). Diffuse astrocytoma and glioblastoma made up the largest proportion of gliomas (n $=16,28.6 \%)$, followed by pilocytic astrocytoma $(\mathrm{n}=16$, $28.6 \%$ ) (Table 1).

The Dean criteria showed that giant cell glioblastomas had the lowest mean, ADCmin, and ADCmax values and the highest Dean criteria score in all types of glioma while pilocytic astrocytoma had the highest mean of ADCmin and ADCmax value. Pilocytic astrocytoma from low-grade glioma had the lowest Dean criteria score.

Table 2 showed cut off and odds ratio for each examination. An independent t-test identified the association between mean and median, which were independent variables, and tumor type according to histopathology. There was a significant difference between all the mean of ADCmin, ADCmax, and conventional MRI scores between the low-grade and high-grade groups $(p<0.05)$.

\section{Receiver Operating Characteristic (ROC) Curve and Area Under the Curve (AUC)}

In this study, a diagnostic test was carried out by creating a ROC curve. Moreover, the AUC was analyzed to measure whether a variable was optimal as a marker for low- or high-grade gliomas. AUC analysis showed that ADCmin and ADCmax and conventional MRI had optimal value as markers for high-grade gliomas. According to the ROC curve, an optimal cut-off was obtained with the highest sensitivity and specificity of each variable. Glioma grades were categorized based on their optimal cut-off. Data were analyzed with cross-tabulation. From these tables, all diagnostic values for each variable were calculated, including sensitivity, specificity, accuracy, PPV, NPV, LR+, and LR-. Figure 4 showed three ROC curves, which were constituted of the ROC curve of separated examination, ROC curve of two-parameter combination, and ROC curve 

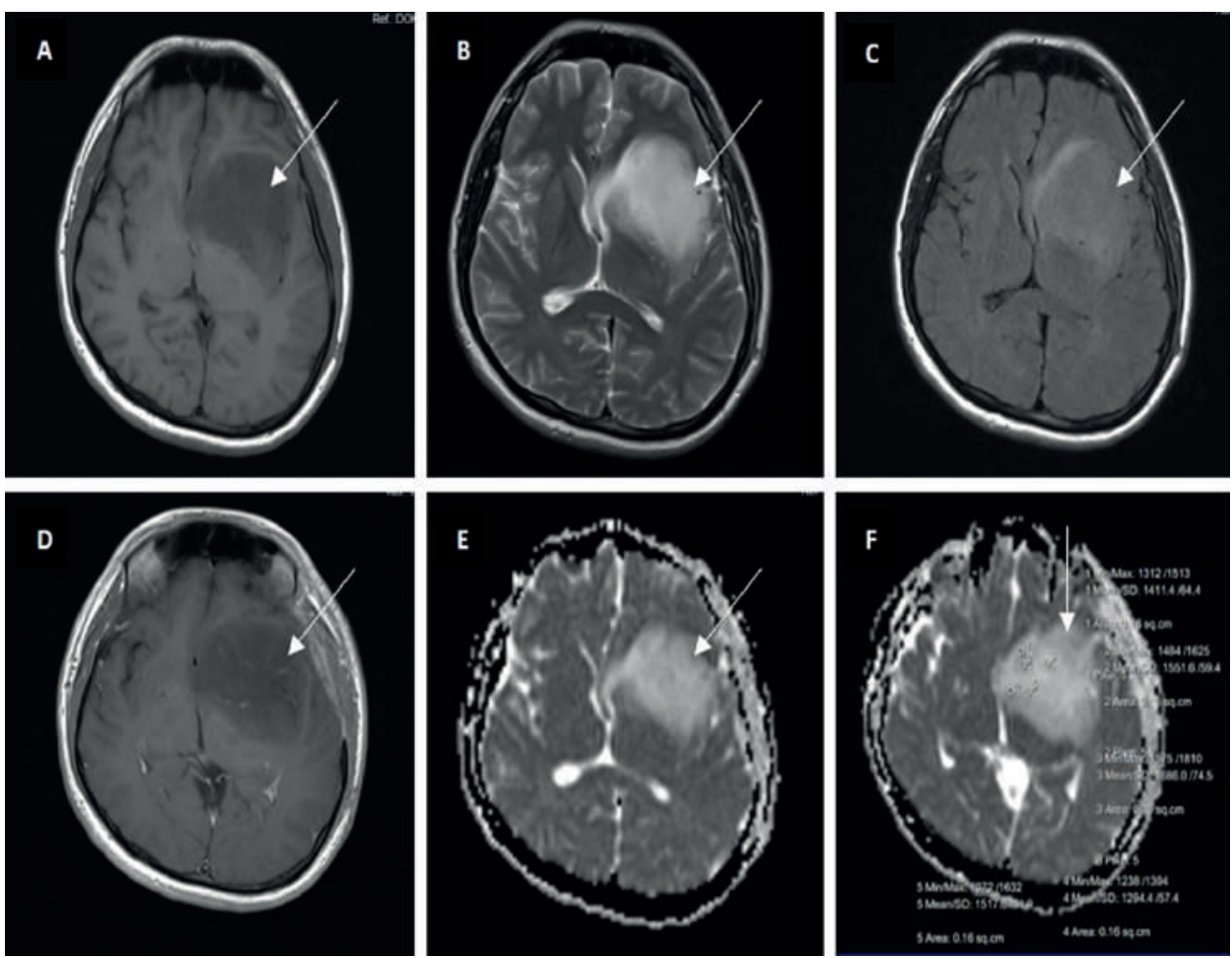

Figure 1. Low grade glioma (WHO Grade II). A: T1-weighted image (T1WI); B: T2-weighted image (T2WI); C: Fluid-Attenuated Inversion Recovery (FLAIR); D: T1WI-post contrast; E: diffusion-weighted imaging (DWI); F: tumoral apparent diffusion coefficient (ADC).
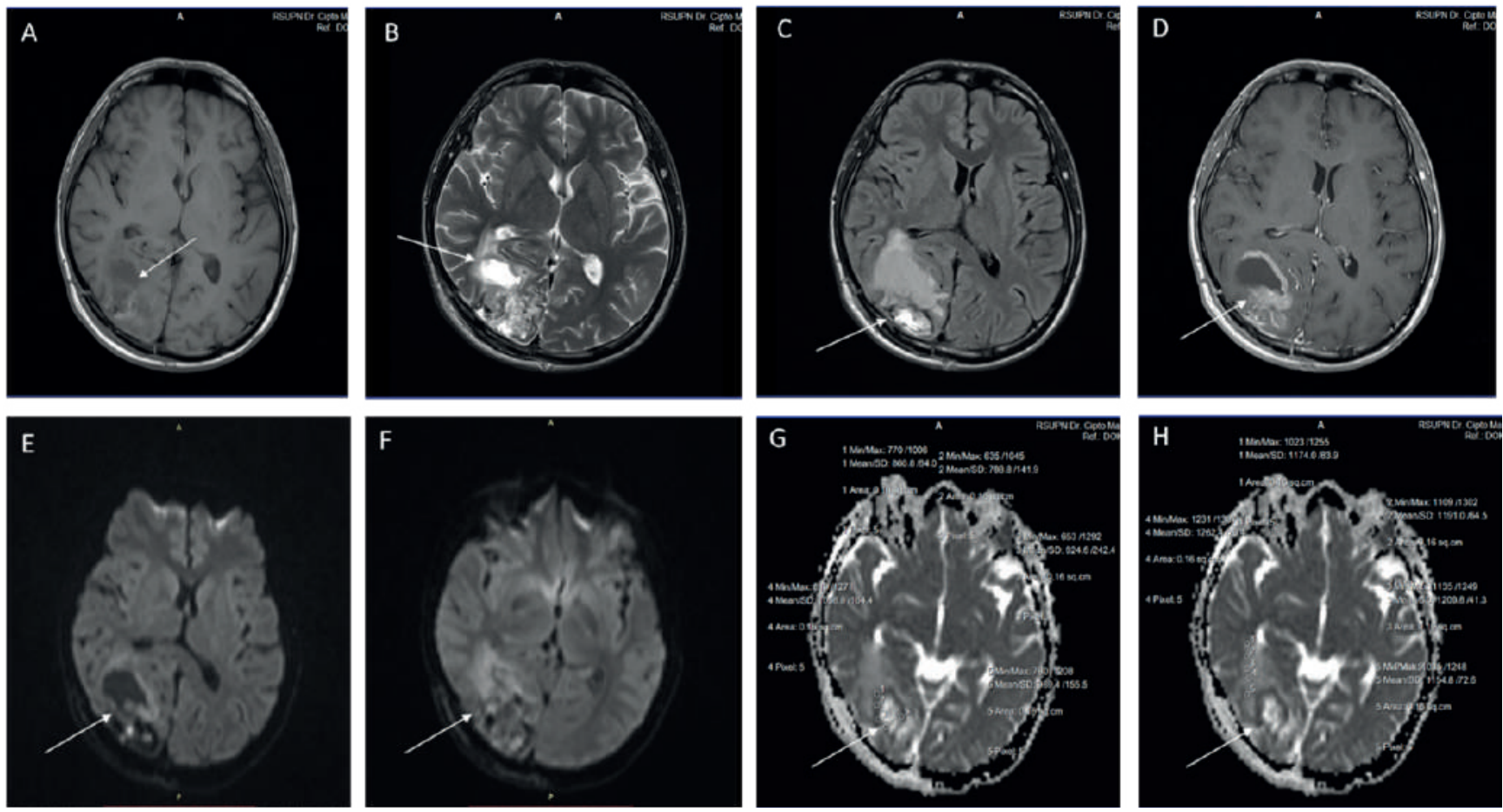

Figure 2. High grade glioma (WHO Grade III). A: T1-weighted image (T1WI); B: T2-weighted image (T2WI); C: Fluid-Attenuated Inversion Recovery (FLAIR); D: T1WI-post contrast; E: diffusion-weighted imaging (DWI); F: DWI; G: tumoral apparent diffusion coefficient (ADC); H: peritumoral ADC. 

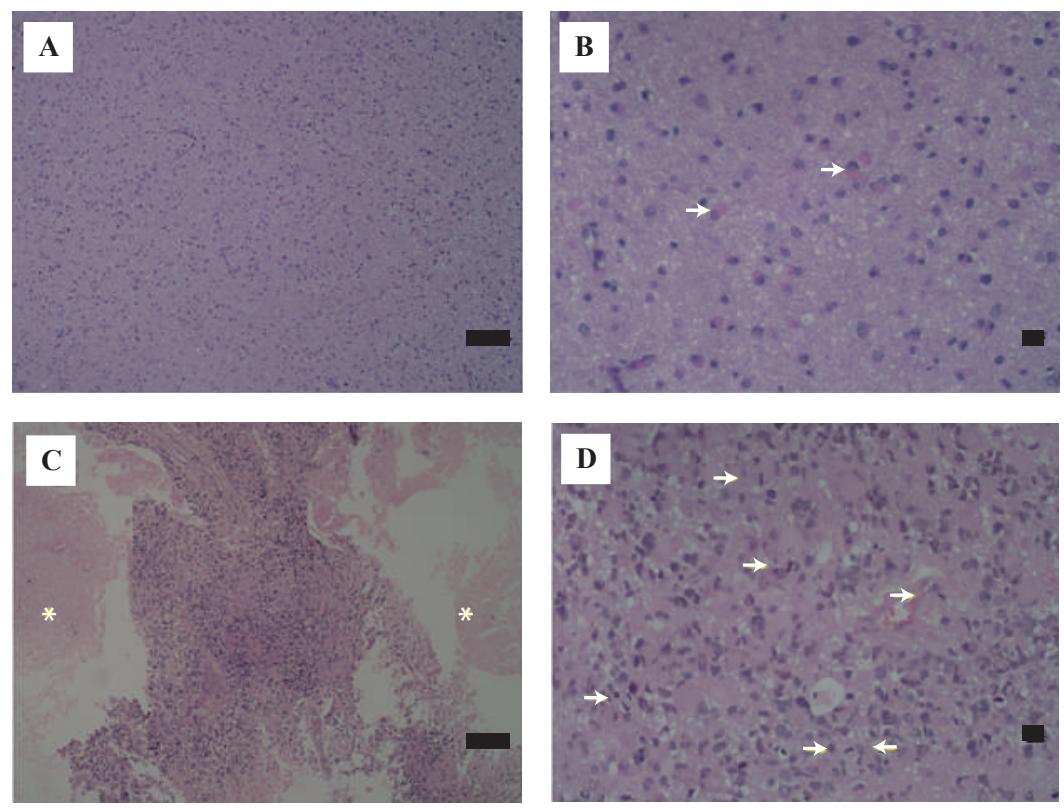

Figure 3. The histopathological findings of diffuse astrocytoma (WHO Grade II) and glioblastoma (WHO Grade IV) based on hematoxylin and eosin staining result. A: diffuse astrocytoma, moderately cellular tumor consist of proliferating neoplastic astrocytes; B: diffuse astrocytoma, some of tumor cells show gemistocytic morphology with abundant glassy eosinophilic cytoplasm (arrow); C: glioblastoma, moderate to highly cellular tumor (middle) with areas of necrosis (asterisks); D: glioblastoma, tumor cells show astrocytic differentiation, some with gemistocytic morphology and brisk mitotic figures (arrow). of three-parameter combination. From these ROC curves, AUC values for low-grade glioma were greater than the reference line $(50 \%)$ with the highest AUC from separated examination at $80.4 \%(p<0.0001 ; 95 \% \mathrm{CI}: 68.2-92.5 \%)$ and space-occupying combination at $78.6 \%(p<0.0001 ; 95 \% \mathrm{CI}$ : $66.1-91.1 \%$ ), and three-parameter combination at $78.6 \%$ ( $p=0.001,95 \%$ : CI 63.9-89.7\%) (Table 3).

The ROC of the three-parameter combination had a higher peak for sensitivity and specificity compared to a single examination. However, exact calculation and crosstabulation have been executed to determine the diagnostic properties of each examination. Both separated and combined examination had ROCs that were above the reference line (50\%), which indicated that both examinations had good potential for glioma diagnostic and grading examination.

From Table 4, it can be seen that all separated or combined examinations have statistically significant AUC values. However, we found that the combined examination did not improve AUC values.

\section{Combinations for Low-Grade Tumor Markers}

Bivariate analysis was carried out in each variable, which met the requirement for multivariate analysis determination $(p<0.25)$. Logistic regression analysis with

Table 1. Glioma classification and variation of examination results $(n=56)$.

\begin{tabular}{|c|c|c|c|c|}
\hline Histology & $\begin{array}{l}\text { Frequency } \\
(\text { (n (\%)) }\end{array}$ & $\begin{array}{c}\text { Mean } \\
\text { Conventional MRI } \\
\text { (Dean Criteria) }\end{array}$ & $\begin{array}{c}\text { Mean ADCmin } \\
\left(\times 10^{-3} \mathrm{~mm}^{2} / \mathrm{s}\right)\end{array}$ & $\begin{array}{c}\text { Mean ADCmax } \\
\left(\times 10^{-3} \mathrm{~mm}^{2} / \mathrm{s}\right)\end{array}$ \\
\hline \multicolumn{5}{|l|}{ Low-grade Glioma } \\
\hline Diffuse Astrocytoma & $16(28.6)$ & $8.7 \pm 2.8$ & $1058 \pm 452$ & $1310 \pm 394.4$ \\
\hline Pilocytic Astrocytoma & $9(16.1)$ & $6.6 \pm 3.4$ & $1088.2 \pm 286$ & $1354.8 \pm 306.8$ \\
\hline Pleiomorphic Xanthoastrocytoma & $2(3.6)$ & $11 \pm 1.4$ & $857 \pm 173.9$ & $1292 \pm 65.8$ \\
\hline Oligondendroglioma, NOS & $1(1.8)$ & 9 & 786 & 1033 \\
\hline \multicolumn{5}{|l|}{ High-grade Glioma } \\
\hline Glioblastoma & $16(28.6)$ & $10.4 \pm 1.9$ & $621.5 \pm 248.2$ & $851.6 \pm 254.3$ \\
\hline Anaplastic Astrocytoma & $4(7.1)$ & $11.25 \pm 1.3$ & $644 \pm 292.6$ & $937.5 \pm 316.3$ \\
\hline Anaplastic Oligodendroglioma & $4(7.1)$ & $8 \pm 2.4$ & $834 \pm 147$ & $976 \pm 210.4$ \\
\hline Anaplastic Oligoastrocytoma & $3(5.4)$ & $7 \pm 2$ & $752.7 \pm 147.0$ & $988 \pm 210.4$ \\
\hline Giant Cell Glioblastoma & $1(1.8)$ & 12 & 361 & 503 \\
\hline
\end{tabular}

ADC: apparent diffusion coefficient; ADCmin: minimum ADC; ADCmax: maximum ADC, MRI: magnetic resonance imaging; NOS: not-otherwise-specified. 
Table 2. Cut off and odds ratio for each examination.

\begin{tabular}{|c|c|c|c|c|c|}
\hline \multirow[b]{2}{*}{ Cut-off } & & \multicolumn{2}{|c|}{ Glioma } & \multirow[b]{2}{*}{ OR $(95 \%$ CI $)$} & \multirow[b]{2}{*}{$\operatorname{aOR}(95 \% \mathrm{CI})$} \\
\hline & & $\begin{array}{c}\text { High } \\
(\mathbf{n}(\%))\end{array}$ & $\begin{array}{c}\text { Low } \\
(\mathbf{n}(\%))\end{array}$ & & \\
\hline \multirow[t]{2}{*}{ cMRI } & $>9.5$ & $17(60.7)$ & $8(28.6)$ & \multirow{2}{*}{$3.8(1.2-11.8)$} & \multirow{2}{*}{$(-)$} \\
\hline & $\leq 9.5$ & 11(39.3) & $20(71.4)$ & & \\
\hline \multirow[t]{2}{*}{$\mathrm{ADC} \min \left(\times 10^{-3} \mathrm{~mm}^{2} / \mathrm{s}\right)$} & $\leq 888.5$ & $21(77)$ & $6(22)$ & \multirow{2}{*}{$(-)$} & \multirow{2}{*}{$11(3.2-38.5)$} \\
\hline & $>888.5$ & $7(24)$ & $22(76)$ & & \\
\hline \multirow[t]{2}{*}{$\mathrm{ADC} \max \left(\mathrm{x} 10^{-3} \mathrm{~mm}^{2} / \mathrm{s}\right)$} & $\leq 1085.5$ & $22(78)$ & $5(18)$ & \multirow{2}{*}{$(-)$} & \multirow{2}{*}{$16(4.5-63.3)$} \\
\hline & $>1085.5$ & $6(21)$ & $23(82)$ & & \\
\hline
\end{tabular}

OR: odds ratio; 95\% CI: 95\% confidence interval; aOR: adjusted odds ratio.

a stepwise backward method was done. However, neither variable meets the required $p$-value $(p \leq 0.05)$. Therefore, the diagnostic value could only be analyzed using crosstabulation for the combined examination variables. Lowand high-grade tumor diagnoses were categorized with combinations of markers. The single examination cMRI can diagnosed 17 subjects for low-grade gliomas and 20 subjects for high-grade glioma. Whereas a combination of cMRI and ADC examination can diagnose 26 subjects of low-grade gliomas and 20 subjects for high-grade gliomas. These were based on conjunction $(\Lambda$ : AND) and disjunction (V: OR) of logical operation between two and
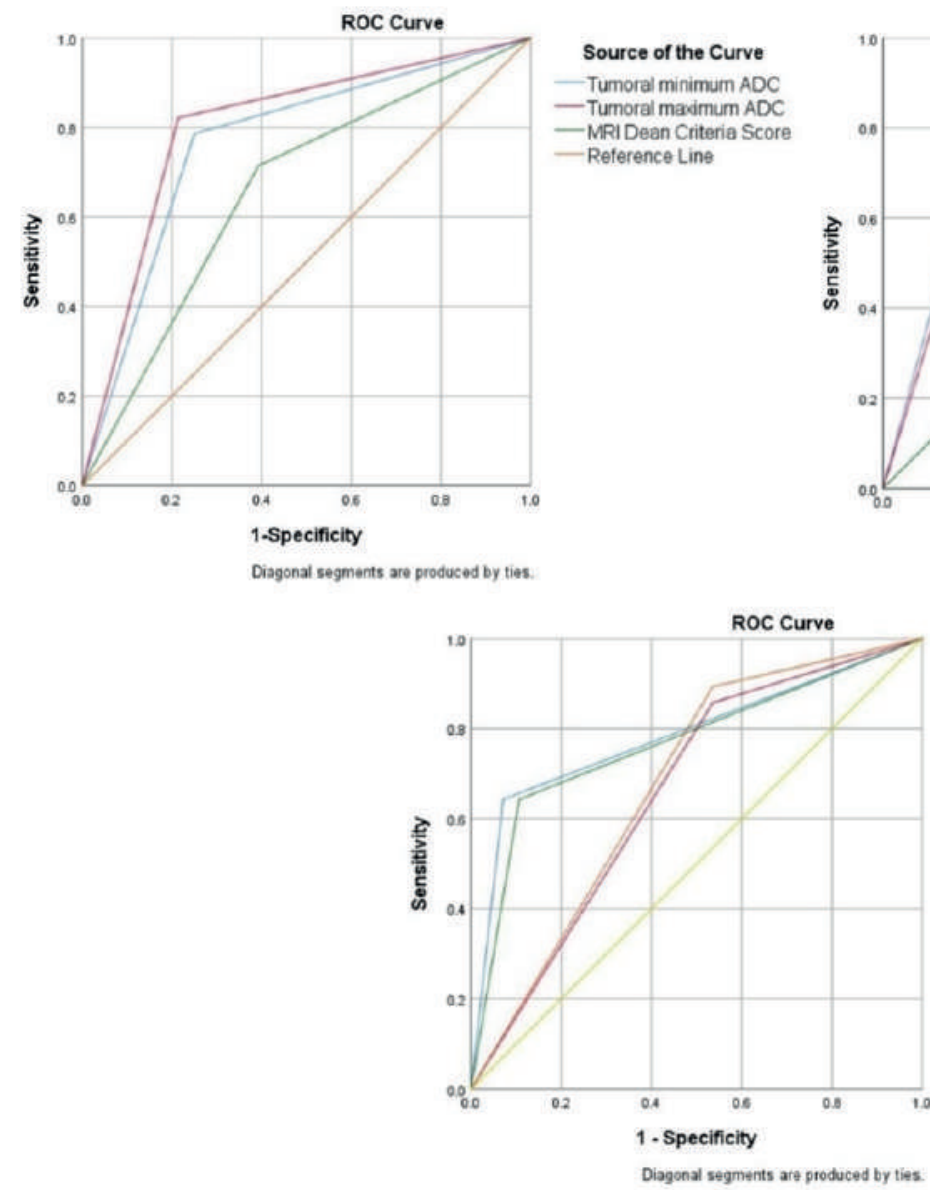

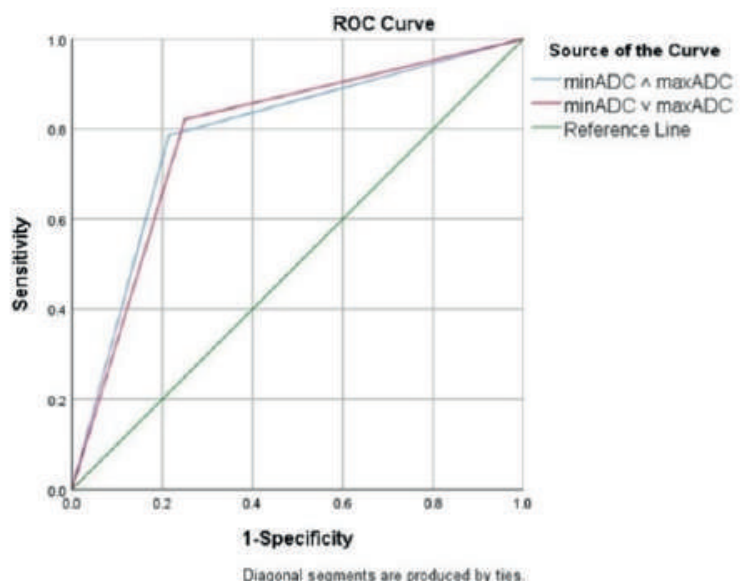

Source of the Curve $-\min A D C \wedge \max A D C \wedge C M R I$ - minADC $\wedge$ maxADC $\vee C M R I$ - $\min A D C \vee \max A D C \wedge$ CMRI - minADC $\vee$ maxADC v CMRI

Reference Line

Figure 4. The receiver operating curve (ROC) of separated examination and combined examination as a parameter for high-grade glioma. 
Table 3. Proportion comparison in low-grade and high-grade glioma.

\begin{tabular}{lccc}
\hline \multicolumn{1}{c}{ Variable } & Mean \pm SD & Median (Min-Max) & p-value* \\
\hline cMRI & & & \\
Total & $9.0 \pm 2.8$ & $10(1-13)$ & 0.022 \\
Low-grade & $8.2 \pm 3.1$ & $9(1-13)$ & \\
High Grade & $9.9 \pm 2.3$ & $10(5-13)$ & \\
\hline ADCmin $\left(\mathbf{x} 1 \mathbf{1 0}^{-3} \mathbf{m m}^{\mathbf{2}} / \mathbf{s}\right)$ & & & \\
Total & $851.7 \pm 369.1$ & $858(103-1907)$ & \\
Low-grade & $1043.7 \pm 376.5$ & $972.5(103-1907)$ & \\
High Grade & $659.8 \pm 243.6$ & $614.5(343-1214)$ & \\
\hline ADCmax $\left(\mathbf{x 1 0} \mathbf{~ m m}^{\mathbf{2}} / \mathbf{s}\right)$ & & & \\
Total & $1098.5 \pm 367.6$ & $1059(503-2227)$ & 0.000 \\
Low-grade & $1313.2 \pm 343.5$ & $1295(666-2227)$ & \\
High Grade & $883.9 \pm 248.3$ & $903 . f 5(503-1419)$ & \\
\hline
\end{tabular}

*Unpaired t-test between low- and high-grade glioma for each variable. cMRI: conventional magnetic resonance imaging; ADCmin: minimum apparent diffusion coefficient (ADC); ADCmax: maximum ADC.

three different variables. Cross-tabulation determined the diagnostic values by comparing histopathological grading according to the WHO Classification as the gold standard to various parameters, including the combination of ADCmin, ADCmax, and conventional MRI as the test variables.

The combination of (ADCmin $\Lambda$ ADCmax) $\Lambda$ cMRI had the highest sensitivity (90\%), accuracy $(78,6 \%)$ and NPV (92.9\%) and the lowest LR- (0.14) compared to single examination cMRI which showed lowest sensitivity (64.5\%), accuracy (66.1\%) and the highest LR- (0.52). The separated examination. ADCmin V ADCmax indicated that if one marker shows positive results for high-grade glioma, it could be identified as a high-grade glioma. Then, the conjunction combination of (ADCmin V ADCmax) $\Lambda$ cMRI indicated that only if both parameters show positive results for high-grade glioma, it can be identified as highgrade glioma. A combination of (ADCmax V ADCmin) V cMRI had the highest PPV (89.3\%) compared to the separated examination. Among the separated and combined examinations, tumoral ADCmax had the highest specificity (81.5\%), accuracy $(80.36 \%)$, and LR+ (4.28).

The combined examination had the lowest negative likelihood ratio (0.14), which was the nearest value to 0 . The lower the ratio, the higher the probability that a highgrade glioma would not be found in patients without clinical features. Likelihood ratios were not influenced by prevalence and could be used in various population settings. On the other hand, the positive likelihood ratio of the combined examinations group had the highest value at 3.99, which indicates that the diagnosis of high-grade glioma was 3.99 times more likely to happen in a patient with clinical features than it would in patients without the clinical features.

\section{Discussion}

The diagnosis of glioma was categorized according to the WHO classification. In this study, glioblastoma and diffuse astrocytoma accounted for almost a third of all glioma. In a recent study reported that glioblastoma represents $29.16 \%$ of all gliomas.(7) However, this finding was different from the CBTRUS Statistical Report data in the USA from 2011 to 2015. According to the study, the proportions of glioma consists of $56.6 \%$ glioblastoma, $9.5 \%$ diffuse astrocytoma, $6.2 \%$ oligodendroglioma, $5.9 \%$ anaplastic astrocytoma, $5.1 \%$ pilocytic astrocytoma and all other glioma.(2)

Two most important predictors of tumor grade were mass effect and necrosis.(15) It has been reported that conventional MRI is highly accurate at differentiating tumor grade in primary brain tumors. The study involved all primary brain tumors with a glioma proportion of $91.5 \%$ or above. The presence of necrosis and contrast enhancement is the best feature in conventional MRI to predict tumor grade (sensitivity $95.9 \%$, specificity $70 \%$ ).(8)

Even though conventional MRI alone has been widely used as a gold standard non-invasive technique to diagnose brain tumors, it is unreliable for differentiating low-grade and high-grade gliomas. $(4,7,16)$ The use of the Dean criteria alone, however, has some limitations. Necrosis 
Table 4. Area under the curve (AUC) comparison of separated and combined examination.

\begin{tabular}{|c|c|c|c|c|c|c|c|}
\hline Examination & Se & Sp & Acc & PPV & NPV & LR+ & LR- \\
\hline \multicolumn{8}{|l|}{ Single Examination } \\
\hline Conventional MRI & $64.5 \%$ & $68.0 \%$ & $66.1 \%$ & $71.4 \%$ & $60.7 \%$ & 2.02 & 0.52 \\
\hline Tumoral ADCmin & $75.9 \%$ & $77.8 \%$ & $76.8 \%$ & $78.6 \%$ & $75.0 \%$ & 3.41 & 0.31 \\
\hline Tumoral ADCmax & $79.3 \%$ & $81.5 \%$ & $80.4 \%$ & $82.1 \%$ & $78.6 \%$ & 4.28 & 0.25 \\
\hline \multicolumn{8}{|l|}{ Combined Examination } \\
\hline $\mathrm{ADCmin} \wedge \mathrm{ADCmax}$ & $78.6 \%$ & $78.6 \%$ & $78.6 \%$ & $78.6 \%$ & $78.6 \%$ & 3.67 & 0.27 \\
\hline $\mathrm{ADCmin} \vee \mathrm{ADCmax}$ & $76.7 \%$ & $80.8 \%$ & $78.6 \%$ & $82.1 \%$ & $75.0 \%$ & 3.99 & 0.29 \\
\hline$(\mathrm{ADCmin} \wedge \mathrm{ADCmax}) \wedge \mathrm{cMRI}$ & $90.0 \%$ & $72.2 \%$ & $78.6 \%$ & $64.3 \%$ & $92.9 \%$ & 3.24 & 0.14 \\
\hline$(\mathrm{ADCmin} \wedge \mathrm{ADCmax}) \vee \mathrm{cMRI}$ & $61.5 \%$ & $76.5 \%$ & $66.1 \%$ & $85.7 \%$ & $46.4 \%$ & 2.62 & 0.5 \\
\hline$(\mathrm{ADCmin} \vee \mathrm{ADCmax}) \wedge \mathrm{cMRI}$ & $85.7 \%$ & $71.4 \%$ & $76.8 \%$ & $64.3 \%$ & $89.3 \%$ & 3.00 & 0.2 \\
\hline$(\mathrm{ADCmin} \vee \mathrm{ADCmax}) \vee \mathrm{cMRI}$ & $62.5 \%$ & $81.2 \%$ & $67.9 \%$ & $89.3 \%$ & $46.4 \%$ & 3.33 & 0.46 \\
\hline $\mathrm{cMRI} \wedge \mathrm{ADCmin}$ & $60.7 \%$ & $35.7 \%$ & $48.2 \%$ & $60.7 \%$ & $47.6 \%$ & 0.94 & 1.10 \\
\hline $\mathrm{cMRI} \wedge \mathrm{ADCmax}$ & $75.0 \%$ & $32.1 \%$ & $53.0 \%$ & $51.0 \%$ & $56.0 \%$ & 1.11 & 0.78 \\
\hline
\end{tabular}

ADCmin: minimum apparent diffusion coefficient (ADC); ADCmax: maximum ADC: cMRI conventional magnetic resonance imaging; AUC: area under curve; Se: sensitivity; Sp: specificity; Acc: accuracy; PPV: positive predictive value; NPV: negative predictive value; LR+: positive likelihood ratio; LR-: negative likelihood ratio; $\wedge$ : conjunction; $\vee$ : disjuction.

and hemorrhage could be related to signal heterogeneity of the lesion. Mass effect is not necessarily associated with the histological grade. Ill-defined tumor border has also been reported in lower-grade glioma, and a well-defined border has conversely been reported in glioblastoma. (8) The lesion in glioma frequently showed similar contrast enhancement, surrounding edema, and mass effect. Some low-grade lesions might demonstrate mild edema, minimal contrast enhancement, or no mass effect, which can be mistakenly interpreted as high-grade gliomas. It has been reported that $20 \%$ of low-grade gliomas show partial contrast enhancement.(16) Conversely, in another study, almost one-fifth of glioblastomas did not show contrastenhancement.(3)

The ADCmin was significantly lower in the highgrade glioma group than in the low-grade glioma group $(p<0.0001)$. This finding is consistent with previous literatures.(4,7,17-19) However, the cut-off between lowand high-grade glioma and the mean ADC value in each group have been found to be variable. $(7,17,19)$ It has been reported that the cut-off value for mean ADC to discriminate low-grade glioma from high-grade glioma is $1.185 \times 10^{-3}$ $\mathrm{mm}^{2} / \mathrm{s}$, with comparable sensitivity to our findings (97.6\%). (19) The range of the ADCmin value of low- and high-grade groups are similar to the results published by a recent study. $\left(1,470 \pm 0.46 \times 10^{-3} \mathrm{~mm}^{2} / \mathrm{s}\right.$ for low grades and $470 \pm 0.38 \times$ $10^{-3} \mathrm{~mm}^{2} / \mathrm{s}$ for high grades.(17) In previous study reported that the mean and ADCmin values of low-grade gliomas are $678.73 \pm 208.52 \times 10^{-3} \mathrm{~mm}^{2} / \mathrm{s}$ and $373.75 \pm 257.06 \times 10^{-3}$ $\mathrm{mm}^{2} / \mathrm{s}$ respectively.(7) These variations can arise from the difference of both low- and high-grade proportions of all gliomas, difference in sample size, and pathology heterogeneity.

Higher ADC values in lower-grade gliomas represent higher water content in the interstitial spaces. Thus, glioma with higher cellularity shows markedly increased signal intensity on DWI and a significant reduction in ADC values. (4) Even though it is less common in the case of brain imaging, artifacts can be affecting images produced by DWI and ADC. Following standard protocols can overcome these limitations.(7)

The combination of conventional MRI and DWIADC features in glioma grade differentiation has been investigated. This study used ADCmin and ADCmax from DWI and the Dean criteria from conventional MRI. The use of conventional MRI and DWI might provide useful information and be reliable and feasible for glioma grading in a more limited facility setting and developing countries. Many studies have investigated the diagnostic accuracy of conventional MRI combined with multiparametric imaging in discriminating tumor grade. It is reported in these previous studies that multiparametric MRI can add diagnostic value in combination with conventional MRI compared with the use of conventional MRI alone. $(8,15,19,20)$

However, there are still limited studies that have combined only ADC features and conventional MRI. Relative ADC (rADC) has been reported to increase diagnostic accuracy in combination with conventional 
MRI features alone (sensitivity of $95.9 \%$ to $98.9 \%$ and specificity of $70 \%$ to $75.9 \%$ ). The ratio of averaged ADCs between tumors and normal areas determined the relative ADC value.(8) In a contrast-enhancing tumor, the value of ADCmin can add useful information to conventional MRI findings. It has been stated that pilocytic astrocytoma has a cut-off value of $1.5 \times 10^{-3} \mathrm{~mm}^{2} / \mathrm{s}$ for the differentiation of high-grade glioma. This result is slightly different from our findings and is possibly due to the difference in sample size and variables that are analyzed.(18)

\section{Conclusion}

The combination of ADC value and conventional MRI is predictive for high-grade glioma. It was found that compared to separated examination, the combination of conventional MRI and advanced MRI improves sensitivity (to 90\%), PPV (to $89.3 \%$ ), NPV (to $92.9 \%$ ), and negative likelihood ratio (to 0.14 ) in differentiating high-grade glioma from lowgrade glioma.

\section{References}

1. Forst DA, Nahed BV, Loeffler JS, Batchelor TT. Low-grade gliomas. Oncologist. 2014; 19: 403-13.

2. Ostrom QT, Gittleman H, Truitt G, Boscia A, Kruchko C, BarnholtzSloan JS. CBTRUS statistical report: primary brain and other central nervous system tumors diagnosed in the United States in 2011-2015. Neuro Oncol. 2018; 20 (Suppl 4): iv1--iv86.

3. Law M, Yang S, Babb JS, Knopp EA, Golfinos JG, Zagzag D, et al. Comparison of cerebral blood volume and vascular permeability from dynamic susceptibility contrast-enhanced perfusion MR imaging with glioma grade. Am J Neuroradiol. 2004; 25: 746-55.

4. Lee EJ, Lee SK, Agid R, Bae JM, Keller A, Terbrugge K. Preoperative grading of presumptive low-grade astrocytomas on MR imaging: diagnostic value of minimum apparent diffusion coefficient. AJNR Am J Neuroradiol. 2008; 29: 1872-7.

5. Ahmed R, Oborski MJ, Hwang M, Lieberman FS, Mountz JM. Malignant gliomas: current perspectives in diagnosis, treatment, and early response assessment using advanced quantitative imaging methods. Cancer Manag Res. 2014; 6: 149-70.

6. Rees JH. Diagnosis and treatment in neuro-oncology: an oncological perspective. Br J Radiol. 2011; 84 (Special Issue 2): S82-9.

7. Darbar A, Waqas M, Enam SF, Mahmood SD. Use of preoperative apparent diffusion coefficients to predict brain tumor grade. Cureus. 2018; 10: e2284. doi: 10.7759/cureus.2284.

8. Guzmán-De-Villoria JA, Mateos-Pérez JM, Fernández-García P, Castro E, Desco M. Added value of advanced over conventional magnetic resonance imaging in grading gliomas and other primary brain tumors. Cancer Imaging. 2014; 14(1): 35. doi: 10.1186/ s40644-014-0035-8.

9. Bulakbasi N, Guvenc II, Onguru O, Erdogan E, Tayfun C, Ucoz T. The added value of the apparent diffusion coefficient calculation to magnetic resonance imaging in the differentiation and grading of malignant brain tumors. Neuro. 2004; 28: 735-46.

10. Nick Bryan R. Introduction: Imaging the brain and its diseases. In: Gillard JH, Waldman AD, Barker PB, editors. Clinical MR Neuroimaging: Physiological and Functional Techniques. 2nd ed. Cambridge: Cambridge University Press; 2010. p.1-4.

11. Baig MA, Klein JP, Mechtler LL. Imaging of brain tumors. Contin Lifelong Learn Neurol. 2016; 22: 1529-52.

12. Bulakbasi N, Kocaoglu M, Örs F, Tayfun C, Ügöz T. Combination of single-voxel proton MR spectroscopy and apparent diffusion coefficient calculation in the evaluation of common brain tumors. Am J Neuroradiol. 2003; 24: 225-33.

13. Kang Y, Choi SH, Kim YJ, Kim KG, Sohn CH, Kim JH, et al. Gliomas: Histogram analysis of apparent diffusion coefficient maps with standard- or high-b-value diffusion-weighted MR imagingcorrelation with tumor grade. Radiology. 2011; 261: 882-90.

14. Komori T. The 2016 WHO classification of tumours of the central nervous system: The major points of revision. Neurol Med Chir. 2017; 57: 301-11.

15. Law M, Yang S, Wang H, Babb JS, Johnson G, Cha S, et al. Glioma grading: sensitivity, specificity, and predictive values of perfusion MR imaging and proton MR spectroscopic imaging compared with conventional MR imaging. AJNR Am J Neuroradiol. 2003; 24 1989-98.

16. Zhang L, Min Z, Tang M, Chen S, Lei X, Zhang X. The utility of diffusion MRI with quantitative ADC measurements for differentiating high-grade from low-grade cerebral gliomas: Evidence from a meta-analysis. J Neurol Sci. 2017; 373: 9-15.

17. Mahdiyeh S, Fariborz F, Hossein G, Mojtaba M, Ayoob R, Soheilah $\mathrm{K}$, et al. Grading of glioma tumors by analysis of minimum apparent diffusion coefficient and maximum relative cerebral blood volume. Casp J Neurol Sci. 2017; 2(4): 42-53.

18. Murakami R, Hirai T, Kitajima M, Fukuoka H, Toya R, Nakamura $\mathrm{H}$, et al. Magnetic resonance imaging of pilocytic astrocytomas: usefulness of the minimum apparent diffusion coefficient (ADC) value for differentiation from high-grade gliomas. Acta radiol. 2008; 49: 462-7.

19. Hilario A, Ramos A, Perez-Nuñez A, Salvador E, Millan JM, Lagares $\mathrm{A}$, et al. The added value of apparent diffusion coefficient to cerebral blood volume in the preoperative grading of diffuse gliomas. Am J Neuroradiol. 2012; 33: 701-7.

20. Zonari P, Baraldi P, Crisi G. Multimodal MRI in the characterization of glial neoplasms: the combined role of single-voxel MR spectroscopy, diffusion imaging and echo-planar perfusion imaging. Neuroradiology. 2007; 49: 795-803. 